OPEN ACCESS

Edited by:

Chonlaphat Sukasem, Mahidol University, Thailand

Reviewed by:

Wei Zhang,

Xiangya Hospital Central South

University, China

Julio Benitez,

University of Extremadura, Spain

*Correspondence:

Junwei Fan

driunweifan@163.com

Zhihai Peng

pengzhihai1958@163.com

${ }^{\dagger}$ These authors have contributed equally to this work

Specialty section:

This article was submitted to

Pharmacogenetics

and Pharmacogenomics,

a section of the journal

Frontiers in Genetics

Received: 19 November 2018

Accepted: 14 May 2019

Published: 31 May 2019

Citation:

Liu Y, Zhang C, Li L, Ou B, Yuan L, Zhang T, Fan J and Peng Z (2019) Genome-Wide Association Study of Tacrolimus Pharmacokinetics Identifies Novel Single Nucleotide Polymorphisms in the Convalescence and Stabilization Periods of Post-transplant Liver Function.

Front. Genet. 10:528.

doi: 10.3389/fgene.2019.00528

\section{Genome-Wide Association Study of Tacrolimus Pharmacokinetics Identifies Novel Single Nucleotide Polymorphisms in the Convalescence and Stabilization Periods of Post-transplant Liver Function}

\author{
Yuan Liu't, Chengdong Zhang't, Lei Li1t, Baochi Ou', Liyun Yuan³, Tao Zhang4, \\ Junwei Fan ${ }^{1 *}$ and Zhihai Peng ${ }^{1 *}$ \\ 'Department of Hepatobiliary Pancreatic Surgery, Shanghai General Hospital, School of Medicine, Shanghai Jiao Tong \\ University, Shanghai, China, ${ }^{2}$ State Key Laboratory of Genetic Engineering, School of Life Sciences, Fudan University, \\ Shanghai, China, ${ }^{3}$ Key Lab of Computational Biology, CAS-MPG Partner Institute for Computational Biology, \\ Shanghai Institutes for Biological Sciences, Chinese Academy of Sciences, Shanghai, China, ${ }^{4}$ Department of Organ \\ Transplant, The Second Affiliated Hospital to Guangzhou Medical University, Guangzhou, China
}

After liver transplantation, the liver function of a patient is gradually restored over a period of time that can be divided into a convalescence period (CP) and a stabilizing period (SP). The plasma concentration of tacrolimus, an immunosuppressant commonly used to prevent organ rejection, varies as a result of variations in its metabolism. The effects of genetic and clinical factors on the plasma concentration of tacrolimus appear to differ in the CP and SP. To establish a model explaining the variation in tacrolimus trough concentration between individuals in the CP and SP, we conducted a retrospective, single-center, discovery study of 115 pairs of patients (115 donors and 115 matched recipients) who had undergone liver transplantation. Donors and recipients were genotyped by a genome-wide association study (GWAS) using an exome chip. Novel exons were identified that influenced tacrolimus trough concentrations and were verified with bootstrap analysis. In donors, two single-nucleotide polymorphisms showed an effect on the CP (rs1927321, rs1057192) and four showed an effect on the SP (rs776746, rs2667662, rs7980521, rs4903096); in recipients, two singlenucleotide polymorphisms showed an effect in the SP (rs7828796, rs776746). Genetic factors played a crucial role in tacrolimus metabolism, accounting for $44.8 \%$ in the SP, which was higher than previously reported. In addition, we found that CYP3A5, which is known to affect the metabolism of tacrolimus, only influenced tacrolimus pharmacokinetics in the SP.

Keywords: GWAS, CYP3A5, exome, tacrolimus, FAM26F 


\section{INTRODUCTION}

Tacrolimus is one of the most common and potent immunosuppressive drugs used to lower the risk of acute organ rejection in patients through the suppression of the cytotoxic activity of the $\mathrm{T}$ lymphocytes involved in acute rejection; however, it has a narrow therapeutic window (Liu et al., 1991; Kelly et al., 2004; Wu et al., 2018). The post-operation blood concentration of tacrolimus should be strictly monitored in accordance with the recommended time-dependent target trough concentrations, as subtherapeutic doses of tacrolimus are associated with acute rejection and graft loss (Rehman et al., 2014) and overdosing of tacrolimus produces adverse side effects, such as nephrotoxicity and new-onset diabetes (Kershner and Fitzsimmons, 1996; Shi et al., 2013; Ling et al., 2017). The metabolism of tacrolimus, which plays an essential role in its blood concentration, is largely dependent on the cytochrome P450 3A (CYP3A) subfamily, especially CYP3A5 (Mourad et al., 2005). As the genetic polymorphisms of CYP3A5 affect its expression and activity, the effects of CYP3A5 polymorphisms on the metabolism of tacrolimus have been studied thoroughly over the last few decades to provide an understanding of the variance in tacrolimus blood concentrations among patients (Alvarez-Elias et al., 2016; Deininger et al., 2016). Patients with the CYP $3 \mathrm{~A} 5 * 3 / * 3$ genotype (non-expressors) receiving orthotopic liver transplantation (OLT) or renal transplantation require a lower tacrolimus dose to reach similar trough levels compared with those with the CYP3A5*1 allele (expressors) (MacPhee et al., 2005; Renders et al., 2007; Argudo et al., 2015; Kato et al., 2016). In addition to CYP3A5, single nucleotide polymorphisms (SNPs) of other genotypes have been implicated in tacrolimus metabolism, including CYP3A4 and recipient $\mathrm{ABCB} 1$ (adenosine triphosphate-binding cassette sub-family B member 1) (Shi et al., 2013; Deininger et al., 2016; Debette-Gratien et al., 2016), although no consensus has been reached. In the present study, considering that multiple unknown genes may be involved in tacrolimus metabolism, we performed a genome-wide association study (GWAS) to evaluate more than 240,000 exonic variants in 115 donors and 115 matched recipients with the associated tacrolimus trough concentrations using an exome chip, and established valid models to account for the variation in tacrolimus concentrations.

\section{MATERIALS AND METHODS}

\section{Study Subjects}

In this study, 115 donors and 115 matched recipients receiving an OLT between July 2015 and March 2017 at the First People's Hospital, affiliated to Shanghai Jiao Tong University School of Medicine, and registered in the China Liver Transplant Registry (CLTR) database, were enrolled. All donors were from donation after brain death. This study was registered in ClinicalTrials.gov and the identification number is NCT02752529. All recipients followed the same postoperation treatment protocol: oral tacrolimus, $0.1 \mathrm{mg} / \mathrm{kg} / \mathrm{day}$; steroids, $0.8-1.0 \mathrm{mg} / \mathrm{kg} /$ day; mycophenolate, $1.5 \mathrm{~g} /$ day; and lamivudine combined with low-dose intramuscular hepatitis B immunoglobulin therapy for anti-viral treatment in recipients with hepatitis B virus-related liver disease. If patients had an acute rejection reaction or renal function insufficiency, we increased or decreased the dose of anti-rejection drugs, as appropriate. The enrollment criteria were: (i) adult patients ( $\geq 18$ years of age), (ii) patients that received a tacrolimusbased immunosuppressive regime. The exclusion criteria were: (i) multiorgan transplant patients; (ii) follow-up time less than 1 month; (iii) incomplete patient data. All recipients had associated follow-up information, including tacrolimus trough concentrations. Patients with pre-operative abnormal renal function were excluded. Informed consent was obtained from all subjects or their direct relatives. The study was approved by the Institutional Review Board and conducted strictly in accordance with the guidelines of the Ethics Committee of the Shanghai First Hospital affiliated with Shanghai Jiao Tong University. The methods were conducted in accordance with the Declaration of Helsinki and its later amendments.

\section{Data Collection}

The individual medical records of each enrolled subject were reviewed and recorded at the time of inclusion in the study. The age, gender, body mass index (BMI), blood type, and HLA mismatch of all included subjects were collected. The clinical laboratory index of recipients before and after operation, including glutamic-pyruvic transaminase (ALT), total bilirubin (TB), creatinine $(\mathrm{Cr})$, hemoglobin $(\mathrm{Hb})$, and direct bilirubin (DB), was measured uniformly in the clinical laboratory of the Shanghai General Hospital, affiliated to Shanghai Jiao Tong University. Tacrolimus trough concentrations were measured before morning administration in the first 4 weeks postoperation. Natural logarithms of tacrolimus dose-normalized tacrolimus trough concentration [ln (TAC $\mathrm{C}_{0} / \mathrm{D}$ ratio)] (nanograms per milliliter per total daily dose in milligrams, $\mathrm{ng} / \mathrm{mL} / \mathrm{mg}$ ) were calculated before applying statistical analysis.

\section{Genotyping and Genome-Wide Association Study (GWAS)}

Liver tissue (20-50 mg) was extracted from each donor and recipient, and tacrolimus trough concentration was measured in the whole blood by using the Pro-TracTMII tacrolimus ELISA kit (Diasorin, Stillwater, MN, United States) with a microparticle enzyme immunoassay (ELx 800NB analyzer, BioTek, Winooski, VT, United States). The genomic DNA was collected by using the AllPrep DNA Mini Kit (QIAGEN, Hilden, Germany). DNA concentration was quantified by using a NanoDrop ND2000 spectrophotometer (NanoDrop Technologies, Wilmington, DE, United States). All DNA samples were amplified in two separate multiplex PCR assays. PCR products were cleaned up using AMPure XP Beads (Beckman Coulter, Pasadena, CA, United States) (Lang et al., 2017). Genotypes of all samples were determined by Infinium Human Exome-12 v1.2 BeasChip accessing > 240,000 exonic variants. The CYP3A5 genotype was 
selected from the Drug Metabolizing Enzymes and Transporters (DMET) chip (Liu et al., 2017).

\section{Statistical Analysis}

Quantitative variables were expressed as the mean \pm standard deviation (SD) or the median and interquartile range (IQR). Categorical variables were presented as values and compared by using Pearson's $\chi^{2}$ test. The minor allele frequency (MAF) was set as 0.1. The Hardy-Weinberg Equilibrium (HWE) test was performed using an appropriate $\chi^{2}$ test. Pairwise $R^{2}$ and $D$-values for linkage disequilibrium were calculated by using SHEsis software ${ }^{1}$. SNPs of raw $P$-value with significance were then analyzed according to intersections between 4 weeks of post-operation data collected separately from donors and recipients. SNPs in these intersections were further verified through univariate and multivariate regression analysis, together with clinical baseline characteristics. The significant variables, as determined by multivariate regression analysis, were used to establish a model that could account for the $\ln \left(\mathrm{TAC} \mathrm{C}_{0} / \mathrm{D}\right.$ ratio) observed in the convalescence period (CP) and the stabilizing period (SP). Genotype data analysis and quality control were performed by using PLINK software (Purcell et al., 2007) $)^{2}$. SPSS version 22.0 (SPSS Inc., Chicago, IL, United States) and GraphPad Prism 7 (GraphPad Prism Software Inc., San Diego, CA, United States) were used to perform statistical analysis. Manhattan plots and Venn diagrams were generated by using R Studio ${ }^{3}$. A two-tailed $P$-value of $<0.05$ was deemed significant.

\section{RESULTS}

\section{Baseline Characteristics}

In the study, 115 donors (114 male and 1 female) and 115 recipients (96 male and 19 female) were enrolled in the study. The ages and BMI of donors were not available. There was no significant difference in gender between donors and recipients $(P=1.0)$. The MELD (model for end stage of liver disease) score of recipients was $11.4 \pm 5.9$. The clinical laboratory indices of recipients pre- and post-operation are detailed in Table 1. The median value of dose-normalized tacrolimus trough concentrations was $5.3 \mathrm{ng} / \mathrm{mL} / \mathrm{mg}$ (IQR: 4.8-5.8 ng/mL/mg) in Weeks $1-2$, and $4.7 \mathrm{ng} / \mathrm{mL} / \mathrm{mg}$ (IQR: $4.3-5.3 \mathrm{ng} / \mathrm{mL} / \mathrm{mg}$ ) in Weeks 3-4.

\section{Genome-Wide Association Study}

GWAS was performed using > 240,000 markers on the 115 patient pairs (115 donors and 115 recipients), evaluating the association between SNPs and the natural log of tacrolimus concentration/dose ratios between donors and recipients in the first 4 weeks after OLT, adjusted for age, donor gender, recipient gender, and BMI. All SNPs were shown in order from chromosome 1 to 23 (excluding the Y chromosome). P-values

\footnotetext{
${ }^{1}$ http://analysis.bio-x.cn/myAnalysis.php

${ }^{2}$ http://www.cog-genomics.org/plink2

${ }^{3}$ https://www.rstudio.com/products/rstudio
}

TABLE 1 | Characteristic of patients.

\begin{tabular}{lc}
\hline & Recipient $(\boldsymbol{n}=\mathbf{1 1 5})$ \\
\hline Age, years (mean $\pm \mathrm{SD}$ ) & $47.3 \pm 9.0$ \\
Gender, male/female, $\mathrm{n}$ & $96 / 19$ \\
BMI, kg/m2, (mean $\pm \mathrm{SD})$ & $23.1 \pm 5.0$ \\
MELD score (mean $\pm \mathrm{SD})$ & $11.4 \pm 5.9$ \\
ALT.pre, U/l, median (IQR) & $18.0(12.0-39.0)$ \\
TB.pre, $\mu \mathrm{mol} / \mathrm{L}$, median (IQR) & $23.0(13.0-48.0)$ \\
Cr.pre, mg/dl, median (IQR) & $60.0(47.0-70.0)$ \\
Hb.pre, g/L, median (IQR) & $107.0(81.0-134.0)$ \\
ALT.post, U/I, median (IQR) & $86.6(62.8-131.8)$ \\
TB.post, $\mu$ mol/L, median (IQR) & $36.5(21.7-63.0)$ \\
Cr.post, $\mu$ mol/L, median (IQR) & $57.4(51.3-74.8)$ \\
Hb.post, g/L, median (IQR) & $97.9(89.3-107.7)$ \\
Tacrolimus dose normalized tacrolimus trough in & $5.3(4.8-5.8)$ \\
weeks 1-2, ng/mL/mg, median (IQR) & \\
Tacrolimus dose normalized tacrolimus trough in & $4.7(4.3-5.3)$ \\
weeks 3-4, ng/mL/mg, median (IQR) &
\end{tabular}

\#, missing; BMI, body mass index; ALT.pre, glutamic-pyruvic transaminase detected before transplant; TB.pre, total bilirubin detected before transplant; Cr.pre, creatinine detected before transplant; Hb.pre, hemoglobin detected before transplant; ALT.post, glutamic-pyruvic transaminase detected after transplant; TB.post, total bilirubin detected after transplant; Cr.post, creatinine detected after transplant; Hb.post, hemoglobin detected after transplant; IQR, interquartile range.

were calculated for all SNPs and were shown in a Manhattan plot (Figure 1). The total number of significant correlative SNPs in donors was 105 in Week 1, 103 in Week 2, 123 in Week 3, and 113 in Week 4; in recipients, the number was 138 in Week 1, 132 in Week 2, 138 in Week 3, and 117 in Week 4 (Supplementary Table S1).

\section{Analysis of Differences in Liver Function in Convalescence and Stabilizing Periods}

The liver function of recipients was followed up over the first 4 weeks post-operation as represented by four indicators: alanine aminotransferase (ALT), aspartate aminotransferase (AST), direct bilirubin (DB), and total bilirubin (TB). All four indicators were higher in the first 2 weeks (the convalescence period of liver function; CP) than in the latter 2 weeks (the stabilizing period of liver function; SP). Significant differences between the two phases were observed in ALT $(P=0.011)$, DB $(P<0.0001)$, and TB $(\mathrm{P}<0.0001)$ levels, and the difference in AST almost reached statistical significance $(P=0.078)$ (Figure 2).

\section{Correlative SNPs Between CP and SP in Donors and Recipients}

A Venn diagram of the correlative SNPs with statistical significance in the GWAS of the CP and SP is shown in Figure 3. The number of intersections in donors was 5 in the $\mathrm{CP}$ (intersection between the red and blue ellipses) and 10 in the SP (intersection between the green and yellow). In recipients, the numbers of intersections in the CP and SP were 4 and 6 , respectively. Specific genotypes are detailed in Tables 2, 3 . 


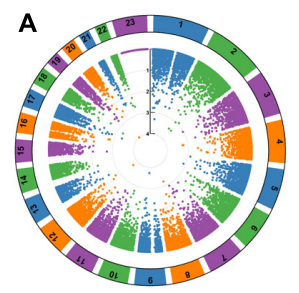

Donor week1

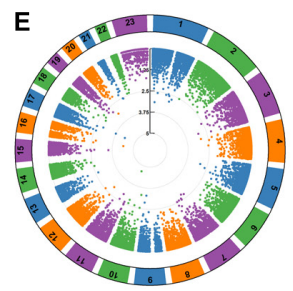

Recipient week1

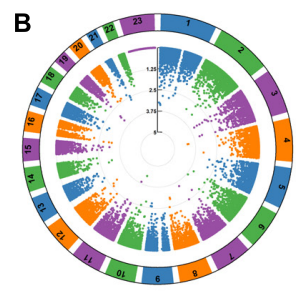

Donor week2

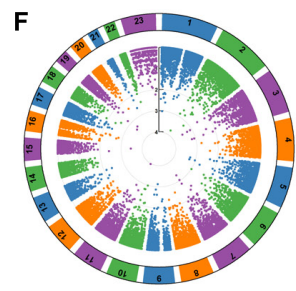

Recipient week2

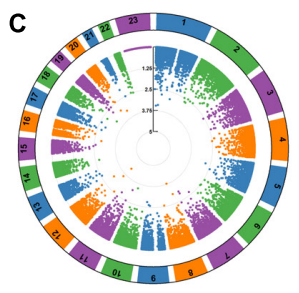

Donor week3

G

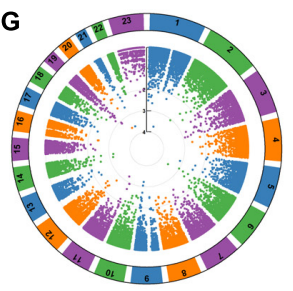

Recipient week3

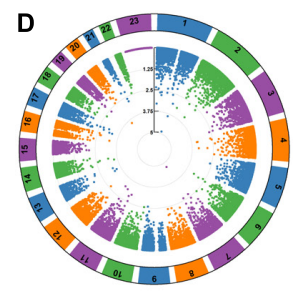

Donor week4

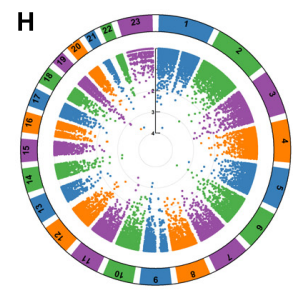

Recipient week4

FIGURE 1 | Manhattan plots of single-nucleotide polymorphisms (SNPS) associated with $\ln \left(T A C \mathrm{C}_{0} / \mathrm{D}\right.$ ratio) in donors and recipients respectively. Total > 240,000 markers were analyzed toward natural log of dose-normalized tacrolimus trough concentrations between donors and recipients in the first 4 weeks with adjustment of age, donor gender, gender and BMI. All SNPs were shown in order from chromosome 1 to 23 (not including Y chromosome). (A) Manhattan plots of single-nucleotide polymorphisms (SNPs) associated with In(TAC $C_{0} / D$ ratio) in donors in week 1. (B) Manhattan plots of single-nucleotide polymorphisms (SNPs) associated with $\ln \left(T A C C_{0} / D\right.$ ratio) in donors in week 2. (C) Manhattan plots of single-nucleotide polymorphisms (SNPs) associated with In(TAC $\mathrm{C}_{0} / \mathrm{D}$ ratio) in donors in week 3. (D) Manhattan plots of single-nucleotide polymorphisms (SNPS) associated with $\ln \left(T A C C_{0} / D\right.$ ratio) in donors in week 4. (E) Manhattan plots of single-nucleotide polymorphisms (SNPs) associated with $\ln \left(T A C C_{0} / D\right.$ ratio) in recipients in week 1. (F) Manhattan plots of single-nucleotide polymorphisms (SNPs) associated with $\operatorname{In}\left(T A C C_{0} / D\right.$ ratio) in recipients in week 2. (G) Manhattan plots of single-nucleotide polymorphisms (SNPs) associated with In(TAC $\mathrm{C}_{0} / \mathrm{D}$ ratio) in recipients in week 3. (H) Manhattan plots of single-nucleotide polymorphisms (SNPs) associated with In(TAC $\mathrm{C}_{0} / \mathrm{D}$ ratio) in recipients in week 4.

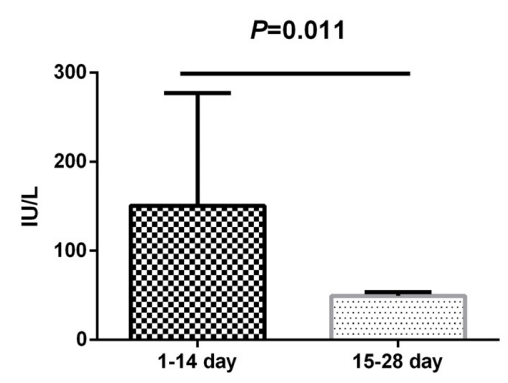

ALT

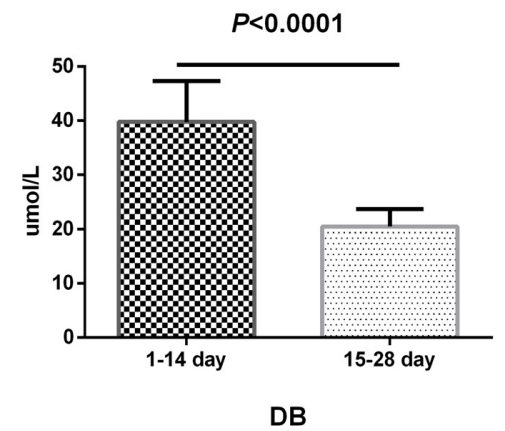

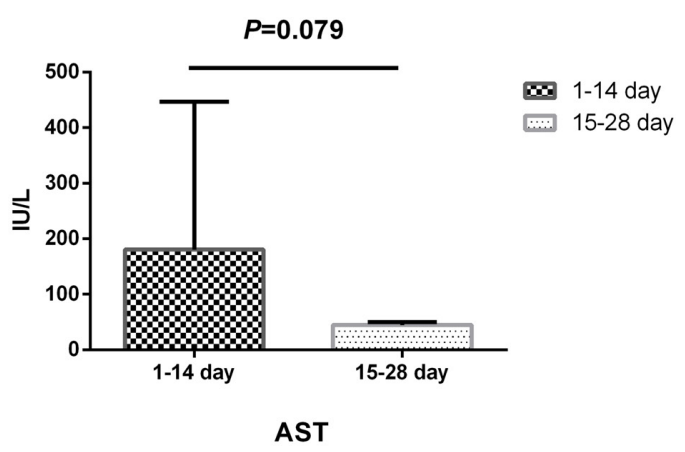

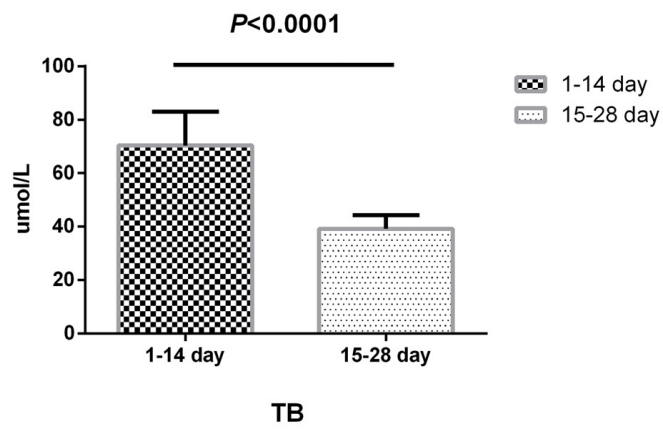

FIGURE 2 | Differences of convalescence phase and stabilizing phase of liver function. ALT, glutamic-pyruvic transaminase; AST, glutamic oxalacetic transaminase; TB, total bilirubin; DB, direct bilirubin. $P<0.05$ was considered significant. 
Donor

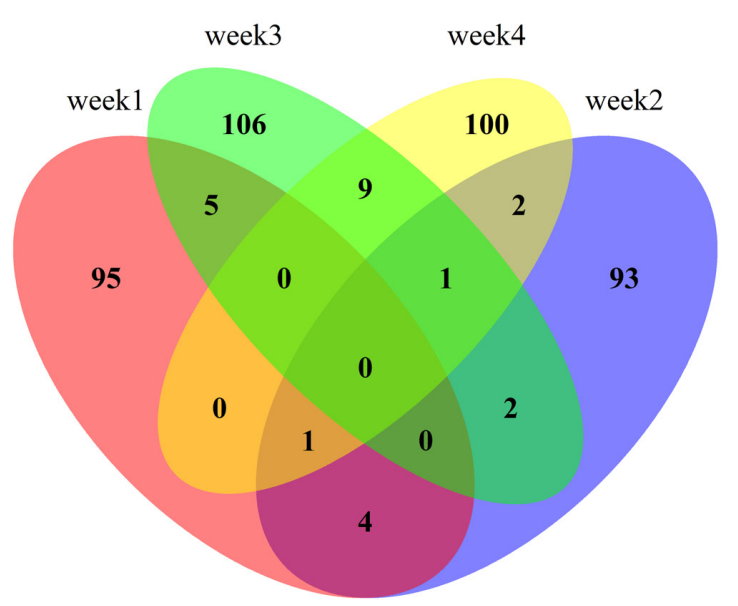

Recipient

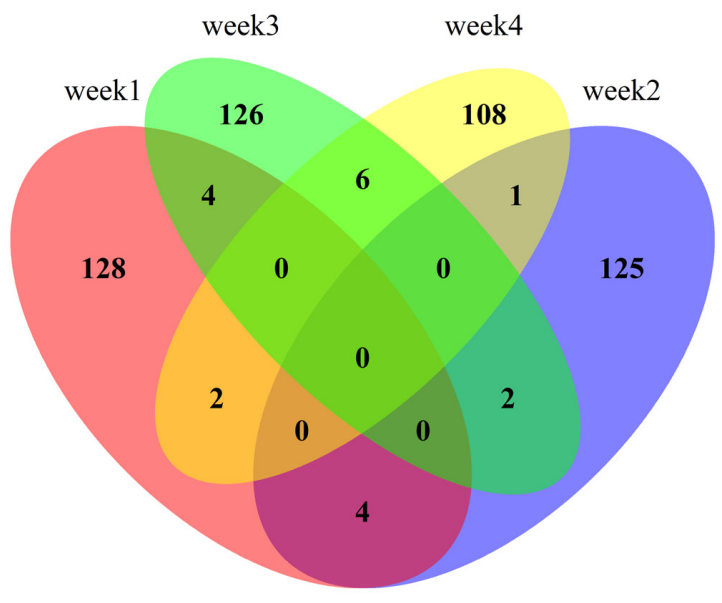

FIGURE 3 | Venn diagram showed the correlation between week1 and week2 (convalescence phase), as well as week3 and week4(stabilizing phase) in donors and recipients respectively. The numbers meant the number of intersections between or among weeks. 0 meant no intersection.

TABLE 2 | Univariate and multivariate regression analysis in convalescence phase.

\begin{tabular}{|c|c|c|c|c|c|c|c|c|c|c|c|c|}
\hline \multirow[b]{3}{*}{ Origin } & \multirow[b]{3}{*}{ Rs } & \multirow[b]{3}{*}{ Gene } & \multirow[b]{3}{*}{ Chr } & \multirow[b]{3}{*}{ Position } & \multicolumn{4}{|c|}{ Univariate } & \multicolumn{4}{|c|}{ Multivariate } \\
\hline & & & & & & & & Bootstrap & & & & Bootstrap \\
\hline & & & & & $\beta$ & $P$-value & Bootstrap $\beta$ & $P$-value & $\beta$ & $P$-value & Bootstrap $\beta$ & $P$-value \\
\hline Donor & rs776746 & CYP3A5 & 7 & 99672916 & 0.286 & 0.008 & 0.286 & 0.008 & 0.194 & 0.058 & 47.733 & 0.062 \\
\hline Donor & rs12677741 & PXDNL & 8 & 52529097 & -0.011 & 0.909 & -0.011 & 0.904 & & & & \\
\hline Donor & rs1058029 & TMX4 & 20 & 7961715 & -0.208 & 0.412 & -0.208 & 0.464 & & & & \\
\hline Donor & rs1927321 & & 9 & $1.21 \mathrm{E}+08$ & 0.310 & 0.001 & 0.310 & 0.007 & 0.233 & 0.008 & 0.233 & 0.015 \\
\hline Donor & rs1827293 & NBPF3 & 1 & 21795388 & -0.158 & 0.078 & -0.158 & 0.073 & & & & \\
\hline Donor & rs1057192 & FAM26F & 6 & 1.17E+08 & -0.345 & 0.000 & -0.345 & 0.001 & -0.289 & 0.001 & -0.289 & 0.001 \\
\hline Recipient & rs11605576 & SLC22A20 & 11 & 64981522 & 0.013 & 0.912 & 0.013 & 0.922 & & & & \\
\hline Recipient & rs11605632 & SLC22A20 & 11 & 64981587 & 0.013 & 0.912 & 0.013 & 0.931 & & & & \\
\hline Recipient & rs12420456 & SLC22A20 & 11 & 64981837 & 0.013 & 0.912 & 0.013 & 0.923 & & & & \\
\hline Recipient & rs3826736 & C19orf45 & 19 & 7571030 & 0.263 & 0.012 & 0.263 & 0.019 & 0.162 & 0.101 & 0.162 & 0.067 \\
\hline Recipient & rs776746 & CYP3A5 & 7 & 99672916 & 0.312 & 0.004 & 0.312 & 0.017 & 0.204 & 0.044 & 0.204 & 0.090 \\
\hline Recipient & Age & & & & 0.000 & 0.968 & 0.000 & 0.979 & & & & \\
\hline Recipient & Gender & & & & -0.069 & 0.704 & -0.069 & 0.673 & & & & \\
\hline Donor & Gender & & & & -0.040 & 0.956 & -0.040 & 0.615 & & & & \\
\hline Recipient & $\mathrm{BMI}$ & & & & 0.027 & 0.295 & 0.027 & 0.295 & & & & \\
\hline Recipient & MELD score & & & & 0.011 & 0.323 & 0.011 & 0.277 & & & & \\
\hline Recipient & ALT.pre & & & & 0.002 & 0.084 & 0.002 & 0.030 & 0.000 & 0.773 & 0.000 & 0.732 \\
\hline Recipient & TB.pre & & & & 0.001 & 0.125 & 0.001 & 0.278 & & & & \\
\hline Recipient & Cr.pre & & & & 0.004 & 0.069 & 0.004 & 0.045 & 0.004 & 0.022 & 0.004 & 0.026 \\
\hline Recipient & Hb.pre & & & & 0.001 & 0.707 & 0.001 & 0.663 & & & & \\
\hline
\end{tabular}

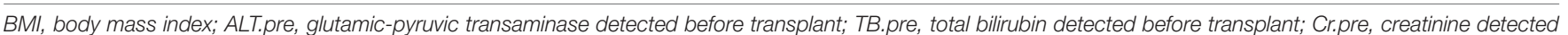
before transplant; Hb.pre, hemoglobin detected before transplant. Bold values indicate the significant level at $P<0.05$.

\section{Analysis of Correlative SNPs and Clinical Variables in Donors and Recipients and Their Relationship With In(TAC C 0 /D Ratio) in the CP and SP}

The association of the correlative SNPs of donors and recipients with $\ln \left(\mathrm{TAC} \mathrm{C}_{0} / \mathrm{D}\right.$ ratio) was explored by univariate and multivariate analyses in the $\mathrm{CP}$ and SP. Clinical variables were simultaneously applied to the regression analysis. The significant variables, as identified by univariate analysis, were then applied to multivariate analysis. The results of the multivariate analysis revealed that there were three significant variables linked to tacrolimus metabolism in CP (Table 2): donor rs1927321 $(P=0.008$, bootstrap $P=0.015)$, donor rs1057192 $(P=0.001$, 
TABLE 3 | Univariate and multivariate regression analysis in stabilizing phase.

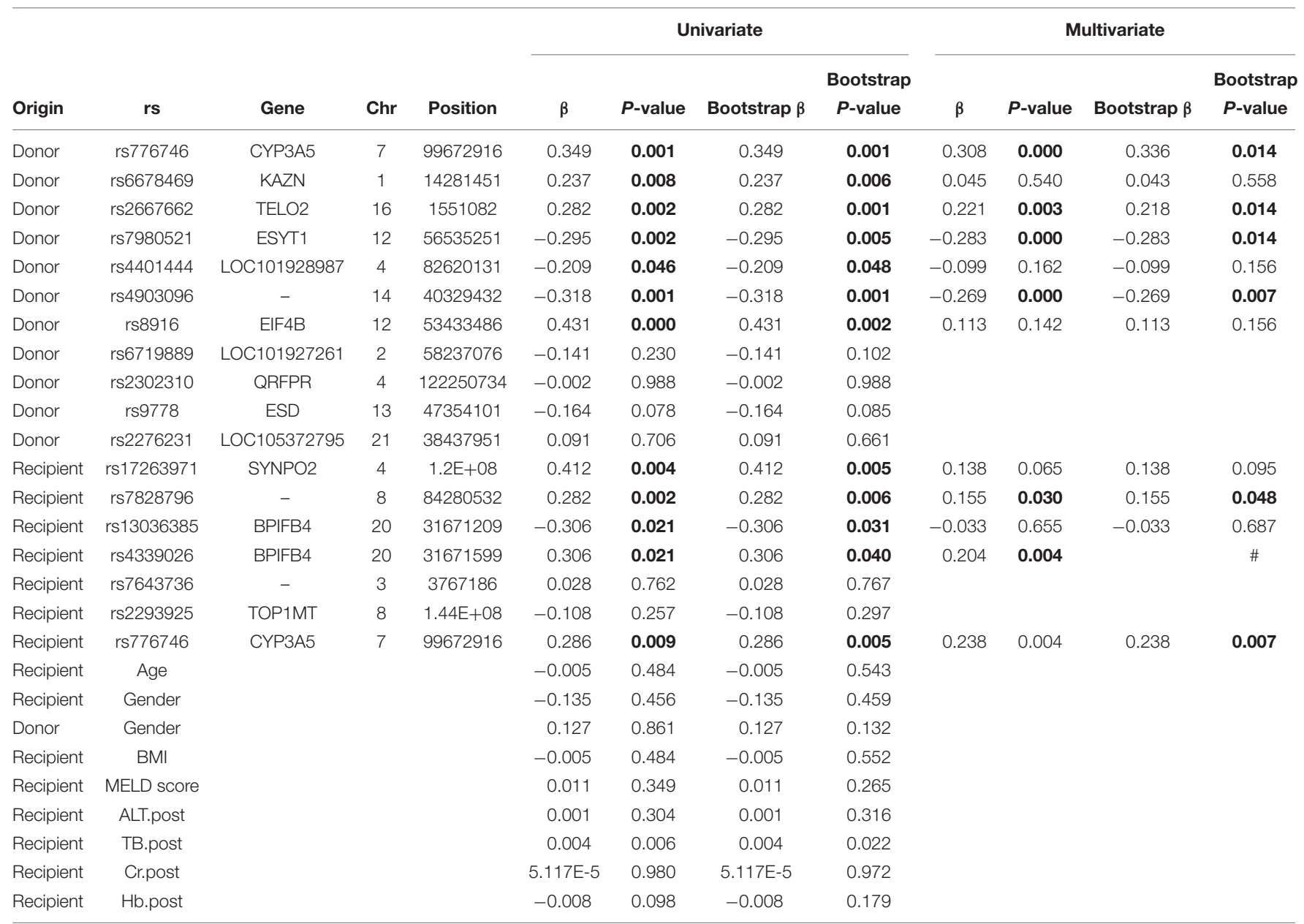

BMI, body mass index; ALT.post, glutamic-pyruvic transaminase detected after transplant; TB.post, total bilirubin detected after transplant; Cr.post, creatinine detected after transplant; Hb.post, hemoglobin detected after transplant. Bold values indicate the significant level at $P<0.05$.

bootstrap $P=0.001)$, and preoperative creatine $(P=0.022$, bootstrap $P=0.026$ ). In SP (Table 3 ), six significant variables were identified, namely: donor rs776746 $(P=0.000$, bootstrap $P=0.014)$, donor $\operatorname{rs} 2667662(P=0.003$, bootstrap $P=0.014)$, donor $\operatorname{rs7980521}(P=0.000$, bootstrap $P=0.014)$, donor rs4903096 $(P=0.000$, bootstrap $P=0.007)$, recipient rs7828796 $(P=0.030$, bootstrap $P=0.048)$, and recipient rs776746 $(P=0.004$, bootstrap $P=0.007)$.

\section{Model of Variation in Tacrolimus Trough Concentration in the CP and SP}

A model was constructed by using significant SNPs and clinical variables to explain variation in tacrolimus trough concentrations in the $\mathrm{CP}$ and SP by linear regression respectively (Table 4). In CP, donor rs1927321 was responsible for $8.9 \%$ variance; donor rs 1057192 was responsible for $11.1 \%$ variance, and Cr.pre was responsible for $2.9 \%$ variance. In SP, donor rs776746, donor rs2667662, donor rs7980521, donor rs4903096, recipient rs7828796, recipient rs776746 were responsible for $10.3,8.2,8.5,10.3,8.1$, and $6.0 \%$ variance, respectively.
Overall, the proportion of variation explained by this model was $22.0 \%$ in CP (adjusted $R^{2}=19.9 \%$ ) and $47.8 \%$ in SP (adjusted $R^{2}=44.8 \%$ ).

\section{DISCUSSION}

Liver transplantation is a major surgical operation, and the liver and gastrointestinal tract of post-operative recipients needs a recovery period to achieve a return to normal physiological functions. Liver function did not recover in the early period after liver transplant (CP), as at that time, the donor liver could not completely metabolize tacrolimus and the genetic function could not be observed. After the donor liver function recovered (in the SP), Tacrolimus could be metabolized completely, which indicated the donors' genetic function. During these two different periods, the genetic and clinical factors affecting tacrolimus metabolism may change. We used an exon microarray in addition to measurements of pre- and post-operative clinical indicators to explore the factors 
TABLE 4 | Tacrolimus variance in natural log-transformed dose-normalized tacrolimus troughs explained by a model in recovery phase and stabilizing phase respectively.

\begin{tabular}{|c|c|c|}
\hline \multirow[t]{2}{*}{ Model } & \multicolumn{2}{|c|}{$\begin{array}{l}\text { Tacrolimus variation } \\
\text { explained by model, \% }\end{array}$} \\
\hline & $R^{2}$ & Adjusted $R^{2}$ \\
\hline \multicolumn{3}{|l|}{ Recovery phase } \\
\hline D rs1927321 & 8.9 & 8.1 \\
\hline D rs1057192 & 11.1 & 10.3 \\
\hline Cr.pre & 2.9 & 2.1 \\
\hline Cr.pre+ D rs1927321 and D rs1057192 & 22.0 & 19.9 \\
\hline \multicolumn{3}{|l|}{ Stabilizing phase } \\
\hline D rs776746 & 10.3 & 9.5 \\
\hline D rs2667662 & 8.2 & 7.3 \\
\hline D rs7980521 & 8.5 & 7.7 \\
\hline D rs4903096 & 10.3 & 9.5 \\
\hline R rs7828796 & 8.1 & 7.3 \\
\hline R rs776746 & 6.0 & 5.1 \\
\hline $\begin{array}{l}\text { D rs776746, D rs2667662, D rs7980521, D } \\
\text { rs4903096, R rs7828796, and R rs776746 }\end{array}$ & 47.8 & 44.8 \\
\hline
\end{tabular}

D meant single nucleotide polymorphisms (SNPS) originated from a donor, R meant single nucleotide polymorphisms (SNPS) originated from the recipient; Cr.pre, creatinine detected before transplant.

influencing tacrolimus metabolism in the different periods of recovery after liver transplantation and used this information to build appropriate models. In addition, we selected the genotype of CYP3A5, known to have a significant effect on tacrolimus metabolism, from the DMET chip. Our study identified two novel SNPs (rs1927321, rs1057192) in donors in the CP, four novel SNPs (rs776746, rs2667662, rs7980521, rs4903096) in donors in the CP, and two novel SNPs (rs7828796, rs776746) in recipients in the $\mathrm{SP}$.

The CYP3A5 genotype is a well-known factor in tacrolimus metabolism in vitro and in vivo, and is currently the most reliable predictor of an individual's tacrolimus dose requirement (Kamdem et al., 2005; Birdwell et al., 2015). As the metabolism of tacrolimus by CYP3A5 occurs mainly in the liver and intestine, CYP3A5 genotypes are relevant in both the donor liver allografts and the recipient native intestines (Masuda and Inui, 2006). Ji et al. (2012) reported that the donor CYP3A5 genotype had a minimal influence on tacrolimus metabolism in CYP3A5 non-expressor recipients during the first month, and that the effect gradually changed over time. In our study, we found that CYP3A5 polymorphisms did not correlate with variations in the tacrolimus trough concentration in either donors or recipients and was thus excluded from the model in the CP. During the SP, the association became more prominent, which indicated that the CYP3A5 polymorphism of donors and recipients did exert a short-term influence on tacrolimus metabolism in the short term, and that this influence amplified over time and may be even more remarkable in the long term.

Our findings of several novel SNPs as independent relevant factors affecting tacrolimus metabolism have not been previously described; these SNPs and their effects may provide new insights into the direction of future research. Among these SNPs, rs266762 is located in TELO2 (telomere maintenance 2), a gene involved in the maintenance of telomere length (You et al., 2016). The TELO2 protein can interact with phosphatidylinositol 3-kinase-related protein kinases and is a component of mTOR (mammalian target of rapamycin) (Fernandez-Saiz et al., 2013). mTOR can protect the liver from ischemia- or reperfusioninduced injury through the NF-kB pathway. Zhang et al. (2017) reported that NF-kB suppressed the expression of PXR (pregnane $\mathrm{X}$ receptor)-mediated CYP3A5 gene. Hence, TELO2 rs26672 might affect tacrolimus pharmacokinetics through the mTOR/NF-kB/CYP3A5 pathway. ESYT1 (Extended synaptotagmin 1) rs7980521 is located on chromosome 12 with major allele G. ESYT1 is an endoplasmic reticulum protein that binds to the plasma membrane and transports lipids (Bian et al., 2018). We supposed that ESYT1 rs7980521 could affect tacrolimus metabolism through lipid transportation because tacrolimus is a fat-soluble drug. Rs1057192 is located in FAM26F (gene family with sequence similarity 26 , member F), a recently identified gene reported to be involved in manifold immune responses (Malik et al., 2017). Interestingly, FAM26F has been found in several gene signatures associated with oxidative stress and inflammation in the study of liver ischemia-reperfusion injury in liver transplantation (Defamie et al., 2008). In our final model, SNPs within FAM26F accounted for 11.2\%, the largest share, of the tacrolimus trough variation in the CP. Therefore, FAM26F may play an intriguing role in liver transplantation and further investigation into the associated genotypes may yield meaningful results.

The influence of other covariates (such as demographics, clinical laboratory indices, disease-related factors, and cotreatments) on tacrolimus metabolism has been extensively investigated (Venkataramanan et al., 1995; Campagne et al., 2018). In our study, we included some frequently reviewed covariates, including age, sex, BMI, MELD score, hematocrit, liver function (levels of AST, ALT, DB, and TB), and serum creatinine. Among all the covariates we studied, pre-operation serum creatinine concentration of recipients in the CP was the only variable that showed statistical significance. In keeping with our findings, serum creatinine was previously shown to be relevant in tacrolimus metabolism (Wang et al., 2017), whereas the other baseline characteristics we studied have been reported to have no effect. Interestingly, serum creatinine only influenced tacrolimus metabolism during $\mathrm{CP}$, showing that, to a certain extent, the major factors affecting tacrolimus metabolism were genetic. The proportion of variation in metabolism explained by our model was $22.0 \%$ in the CP and $47.8 \%$ in the SP, which is, to the best of our knowledge, the highest variation reached (Oetting et al., 2016). This suggested that the effects of genetic factors may be weak or may not occur in the CP, but become stronger in the SP.

There are, unfortunately, several limitations to our study. First, donor demographic data were limited. Fortunately, the quality of donor livers was strictly assessed to satisfy transplantation standards. Under these conditions, the genetic factors were critical. In addition, we did not perform functional 
testing of the genetic loci as the focus of this article was the identification of key SNPs for further study.

\section{CONCLUSION}

We found that SNPs play a non-negligible role in tacrolimus metabolism, and that their role changed over time with the recovery of liver function. The novel SNPs we identified with effects on tacrolimus metabolism could deliver promising new insights into the study of the relationship between patient genotypes and tacrolimus metabolism. The two-phase models we developed based on genetic polymorphism and clinical covariates accounted for a significant portion of the inter-individual variation in tacrolimus trough concentrations and may contribute to the optimization of individual tacrolimus dosing.

\section{DATA AVAILABILITY}

The datasets generated for this study can be found in the GEO https://www.ncbi.nlm.nih.gov/geo/query/acc.cgi?acc= GSE130068.

\section{ETHICS STATEMENT}

Informed consent was agreed and signed by all subjects or their direct relatives. The study was approved by the Institutional Review Board, and carried out strictly abide by the guidelines of the Ethics Committee of the Shanghai First Hospital Affiliated

\section{REFERENCES}

Alvarez-Elias, A. C., Garcia-Roca, P., Velasquez-Jones, L., Valverde, S., VarelaFascinetto, G., and Medeiros, M. (2016). CYP3A5 Genotype and time to reach tacrolimus therapeutic levels in renal transplant children. Transplant. Proc. 48, 631-634. doi: 10.1016/j.transproceed.2016.02.024

Argudo, A., de Aledo Gonzalez, J. M., Alia, P., Ramirez, P., Serrano, T., Fabregat, J., et al. (2015). Liver transplant patient carriers of polymorphism CYP $3 a 5^{*} 1$ donors may need more doses of tacrolimus from the first month after transplantation. Transplant. Proc. 47, 2388-2392. doi: 10.1016/j.transproceed. 2015.09.024

Bian, X., Saheki, Y., and De Camilli, P. (2018). Ca(2+) releases E-Syt1 autoinhibition to couple ER-plasma membrane tethering with lipid transport. EMBO J. 37, 219-234. doi: 10.15252/embj.201797359

Birdwell, K. A., Decker, B., Barbarino, J. M., Peterson, J. F., Stein, C. M., Sadee, W., et al. (2015). Clinical pharmacogenetics implementation consortium (CPIC) guidelines for CYP3A5 genotype and tacrolimus dosing. Clin. Pharmacol. Ther. 98, 19-24. doi: 10.1002/cpt.113

Campagne, O., Mager, D. E., and Tornatore, K. M. (2018). Population pharmacokinetics of tacrolimus in transplant recipients: what did we learn about sources of interindividual variabilities? J. Clin. Pharmacol. 59, 309-325. doi: $10.1002 /$ jcph.1325

Debette-Gratien, M., Woillard, J. B., Picard, N., Sebagh, M., Loustaud-Ratti, V., Sautereau, D., et al. (2016). Influence of donor and recipient CYP3A4, CYP3A5, and ABCB1 genotypes on clinical outcomes and nephrotoxicity in liver transplant recipients. Transplantation 100, 2129-2137.

Defamie, V., Cursio, R., Le Brigand, K., Moreilhon, C., Saint-Paul, M. C., Laurens, M., et al. (2008). Gene expression profiling of human liver transplants identifies
Shanghai Jiao Tong University. The methods were carried out in accordance with the Declaration of Helsinki and its later amendments.

\section{AUTHOR CONTRIBUTIONS}

$\mathrm{YL}$ and $\mathrm{CZ}$ performed all studies. YL and LL drafted the manuscript. YL, BO, and TZ collected the data. YL and LY performed the statistical analysis. ZP and JF participated in the experimental design. All authors read and approved the final manuscript.

\section{FUNDING}

This work was supported by the National Natural Science Foundation of China (Grant No. 8153044) and the Joint Fund of the National Natural Science Foundation of Henan (U1604282 and U1504804). Shanghai three-year action plan to promote clinical skills and clinical innovation capacity of municipal hospitals (No. 16CR2008A).

\section{SUPPLEMENTARY MATERIAL}

The Supplementary Material for this article can be found online at: https://www.frontiersin.org/articles/10.3389/fgene. 2019.00528/full\#supplementary-material

TABLE S1 | SNPs influencing Tacrolimus pharmacikonetics in CP and SP respectively.

an early transcriptional signature associated with initial poor graft function. Am. J. Transplant. 8, 1221-1236. doi: 10.1111/j.1600-6143.2008.02249.x

Deininger, K. M., Vu, A., Page, R. L. II, Ambardekar, A. V., Lindenfeld, J., and Aquilante, C. L. (2016). CYP3A pharmacogenetics and tacrolimus disposition in adult heart transplant recipients. Clin. Transplant. 30, 1074-1081. doi: 10. $1111 /$ ctr. 12790

Fernandez-Saiz, V., Targosz, B. S., Lemeer, S., Eichner, R., Langer, C., Bullinger, L., et al. (2013). SCFFbxo9 and CK2 direct the cellular response to growth factor withdrawal via Tel2/Ttil degradation and promote survival in multiple myeloma. Nat. Cell Biol. 15, 72-81.

Ji, E., Choi, L., Suh, K. S., Cho, J. Y., Han, N., and Oh, J. M. (2012). Combinational effect of intestinal and hepatic CYP3A5 genotypes on tacrolimus pharmacokinetics in recipients of living donor liver transplantation. Transplantation 94, 866-872. doi: 10.1097/TP.0b013e318263700a

Kamdem, L. K., Streit, F., Zanger, U. M., Brockmoller, J., Oellerich, M., Armstrong, V. W., et al. (2005). Contribution of CYP3A5 to the in vitro hepatic clearance of tacrolimus. Clin. Chem. 51, 1374-1381.

Kato, H., Usui, M., Muraki, Y., Tanemura, A., Murata, Y., Kuriyama, N., et al. (2016). Long-term influence of cyp3a5 gene polymorphism on pharmacokinetics of tacrolimus and patient outcome after living donor liver transplantation. Transplant. Proc. 48, 1087-1094. doi: 10.1016/j.transproceed.2016.02.012

Kelly, D., Jara, P., Rodeck, B., Lykavieris, P., Burdelski, M., Becker, M., et al. (2004). Tacrolimus and steroids versus ciclosporin microemulsion, steroids, and azathioprine in children undergoing liver transplantation: randomised European multicentre trial.\%A kelly D. Lancet 364, 1054-1061.

Kershner, R. P., and Fitzsimmons, W. E. (1996). Relationship of FK506 whole blood concentrations and efficacy and toxicity after liver and kidney transplantation. Transplantation 62, 920-926. 
Lang, G. T., Shi, J. X., Hu, X., Zhang, C. H., Shan, L., Song, C. G., et al. (2017). The spectrum of BRCA mutations and characteristics of BRCA-associated breast cancers in China: screening of 2,991 patients and 1,043 controls by next-generation sequencing. Int. J. Cancer 141, 129-142. doi: 10.1002/ijc.30692

Ling, Q., Xie, H., Li, J., Liu, J., Cao, J., Yang, F., et al. (2017). Donor graft microRNAs: a newly identified player in the development of new-onset diabetes after liver transplantation. Am. J. Transplant. 17, 255-264. doi: 10.1111/ajt.13984

Liu, J., Farmer, J. D. Jr., Lane, W. S., Friedman, J., Weissman, I., and Schreiber, S. L. (1991). Calcineurin is a common target of cyclophilin-cycl.osporin A and FKBP-FK506 complexes. Cell 66, 807-815.

Liu, Y., Zhang, T., Li, C., Ye, L., Gu, H., Zhong, L., et al. (2017). SLC28A3 rs7853758 as a new biomarker of tacrolimus elimination and new-onset hypertension in Chinese liver transplantation patients. Biomark. Med. 11, 607-618. doi: 10. 2217/bmm-2017-0128

MacPhee, I. A. M., Fredericks, S., Mohamed, M., Moreton, M., Carter, N. D., Johnston, A., et al. (2005). Tacrolimus Pharmacogenetics: the CYP3A5*1 Allele Predicts Low Dose-Normalized Tacrolimus Blood Concentrations in Whites and South Asians. Transplantation 79, 499-502.

Malik, U., Javed, A., Ali, A., and Asghar, K. (2017). Structural and functional annotation of human FAM26F: a multifaceted protein having a critical role in the immune system. Gene 597, 66-75. doi: 10.1016/j.gene.2016.10.029

Masuda, S., and Inui, K. (2006). An up-date review on individualized dosage adjustment of calcineurin inhibitors in organ transplant patients. Pharmacol. Ther. 112, 184-198.

Mourad, M., Mourad, G., Wallemacq, P., Garrigue, V., Van Bellingen, C., Van Kerckhove, V., et al. (2005). Sirolimus and tacrolimus trough concentrations and dose requirements after kidney transplantation in relation to CYP3A5 and MDR1 polymorphisms and steroids. Transplantation 80, 977-984.

Oetting, W. S., Schladt, D. P., Guan, W., Miller, M. B., Remmel, R. P., Dorr, C., et al. (2016). Genomewide association study of tacrolimus concentrations in african american kidney transplant recipients identifies multiple CYP3A5 alleles. Am. J. Transplant. 16, 574-582. doi: 10.1111/ajt.13495

Purcell, S., Neale, B., Todd-Brown, K., Thomas, L., Ferreira, M. A., Bender, D., et al. (2007). PLINK: a tool set for whole-genome association and population-based linkage analyses. Am. J. Hum. Genet. 81, 559-575.

Rehman, S., Wen, X., Casey, M. J., Santos, A. H., and Andreoni, K. (2014). Effect of different tacrolimus levels on early outcomes after kidney transplantation. Ann. Transplant. 19, 68-75. doi: 10.12659/AOT.889858
Renders, L., Frisman, M., Ufer, M., Mosyagin, I., Haenisch, S., Ott, U., et al. (2007). CYP3A5 genotype markedly influences the pharmacokinetics of tacrolimus and sirolimus in kidney transplant recipients. Clin. Pharmacol. Ther. 81, 228-234.

Shi, Y., Li, Y., Tang, J., Zhang, J., Zou, Y., Cai, B., et al. (2013). Influence of CYP3A4. CYP3A5 and MDR-1 polymorphisms on tacrolimus pharmacokinetics and early renal dysfunction in liver transplant recipients. Gene 512, 226-231. doi: 10.1016/j.gene.2012.10.048

Venkataramanan, R., Swaminathan, A., Prasad, T., Jain, A., Zuckerman, S., Warty, V., et al. (1995). Clinical pharmacokinetics of tacrolimus. Clin. Pharmacokinet. 29, 404-430.

Wang, J., Li, K., Zhang, X., Teng, D., Ju, M., Jing, Y., et al. (2017). The correlation between the expression of genes involved in drug metabolism and the blood level of tacrolimus in liver transplant receipts. Sci. Rep. 7:3429. doi: 10.1038/ s41598-017-02698-w

Wu, J., Zheng, Z., Chong, Y., Li, X., Pu, L., Tang, Q., et al. (2018). Immune responsive release of tacrolimus to overcome organ transplant rejection. $A d v$. Mater. 30:e1805018.

You, J., Sobreira, N. L., Gable, D. L., Jurgens, J., Grange, D. K., Belnap, N., et al. (2016). A syndromic intellectual disability disorder caused by variants in TELO2, a gene encoding a component of the TTT complex. Am. J. Hum. Genet. 98, 909-918. doi: 10.1016/j.ajhg.2016.03.014

Zhang, T., Liu, Y., Zeng, R., Ling, Q., Wen, P., Fan, J., et al. (2017). Association of donor small ubiquitin-like modifier 4 rs237025 genetic variant with tacrolimus elimination in the early period after liver transplantation. Liver Int. 38, 724-732. doi: 10.1111/liv.13597

Conflict of Interest Statement: The authors declare that the research was conducted in the absence of any commercial or financial relationships that could be construed as a potential conflict of interest.

Copyright (C) 2019 Liu, Zhang, Li, Ou, Yuan, Zhang, Fan and Peng. This is an open-access article distributed under the terms of the Creative Commons Attribution License (CC BY). The use, distribution or reproduction in other forums is permitted, provided the original author(s) and the copyright owner(s) are credited and that the original publication in this journal is cited, in accordance with accepted academic practice. No use, distribution or reproduction is permitted which does not comply with these terms. 